\title{
Bios2mds: an R package for comparing orthologous protein families by metric multidimensional scaling
}

\author{
Julien Pelé ${ }^{1 \dagger}$, Jean-Michel Bécu ${ }^{1 \dagger}$, Hervé Abdi $^{2}$ and Marie Chabbert ${ }^{*}$
}

\begin{abstract}
Background: The distance matrix computed from multiple alignments of homologous sequences is widely used by distance-based phylogenetic methods to provide information on the evolution of protein families. This matrix can also be visualized in a low dimensional space by metric multidimensional scaling (MDS). Applied to protein families, MDS provides information complementary to the information derived from tree-based methods. Moreover, MDS gives a unique opportunity to compare orthologous sequence sets because it can add supplementary elements to a reference space.

Results: The R package bios2mds (from BIOlogical Sequences to MultiDimensional Scaling) has been designed to analyze multiple sequence alignments by MDS. Bios2mds starts with a sequence alignment, builds a matrix of distances between the aligned sequences, and represents this matrix by MDS to visualize a sequence space. This package also offers the possibility of performing K-means clustering in the MDS derived sequence space. Most importantly, bios $2 m d s$ includes a function that projects supplementary elements (a.k.a. "out of sample" elements) onto the space defined by reference or "active" elements. Orthologous sequence sets can thus be compared in a straightforward way. The data analysis and visualization tools have been specifically designed for an easy monitoring of the evolutionary drift of protein sub-families.
\end{abstract}

Conclusions: The bios $2 \mathrm{mds}$ package provides the tools for a complete integrated pipeline aimed at the MDS analysis of multiple sets of orthologous sequences in the R statistical environment. In addition, as the analysis can be carried out from user provided matrices, the projection function can be widely used on any kind of data.

Keywords: Metric multidimensional scaling (MDS), Principal coordinate analysis, R program, Supplementary elements, Evolution, Protein family, Phylogeny

\section{Background}

The multiple alignment of homologous sequences provides important information on the evolution and the sequence-function relationships of protein families. Two types of methods, tree-based or space-based methods, can be used to compare sequences (reviewed in [1]). Both methods depend on a multiple alignment of homologous sequences. Tree methods assume a hierarchical, binary structure of the data to infer phylogenetic relationships. On the other hand, space methods are based

\footnotetext{
* Correspondence: marie.chabbert@univ-angers.fr

${ }^{\dagger}$ Equal contributors

'CNRS UMR 6214 - INSERM 1083, Faculté de Médecine, 3 rue Haute de Reculée, Angers 49045, France

Full list of author information is available at the end of the article
}

on multivariate analysis of a distance matrix between the sequences and do not assume a specific structure for the data. Such a method is metric multidimensional (MDS) which is a powerful method to visualize distances between elements [2-5]. MDS, also named principal coordinate analysis, starts from a matrix of distances between elements and visualizes these elements in a low dimensional space in which the distances best approximate the original distances. Applied to biological sequences, this method usefully complements phylogeny [6-11].

The completion of the genome sequencing of a wide variety of organisms has paved the way to the comparison of protein families from different species. A very interesting property of MDS is the possibility to project supplementary elements onto a reference or "active" 
space. The positions of the supplementary elements (a.k. a. "out of sample" elements) are obtained from their distance to the active elements $[2,12,13]$. This property provides a very useful tool to compare orthologous sequences to a reference sequence set. In particular, when several orthologous protein families are compared, this method can be used to visualize evolutionary drifts [9].

MDS is based on the eigen-decomposition (i.e., principal component analysis) of a cross-product matrix derived from the distance matrix [2-5] and can be performed with the default tools included in the $\mathrm{R}$ statistical language (e.g., cmds function). In addition, several $\mathrm{R}$ packages such as ade4, made4, adegenet, and vegan [1417] have been developed to provide multivariate analysis in the field of bioinformatics, including MDS. For example, the dudi.pca function in ade4 [14] or the wcmdscale function in vegan [17] performs MDS analysis. However, the projection technique has not been widely used yet and, to the best of our knowledge, is not included in the available $R$ packages.

Thus, we have developed the $\mathrm{R}$ package bios $2 m d s$ (from BIOlogical Sequences to MultiDimensional Scaling) to provide all the tools necessary to perform the MDS analysis of multiple sequence alignments. This package includes a function that projects supplementary sequences onto a reference space and, thus, makes it possible to compare orthologous sequence sets.

\section{Implementation \\ Main features}

The bios $2 m d s$ package has been developed in the R statistical environment. $\mathrm{R}$ was chosen because it is opensource, accessible under the GNU General Public License and widely used within the bioinformatics community. $\mathrm{R}$ packages can take advantage of functions already developed in available packages. Here, the aim of the package is to provide the tools necessary to compare orthologous sequence sets by MDS analysis, namely to analyze the active set by metric MDS to define an active space and project supplementary orthologous sequences onto this active space.

While it is possible to use available packages such as ape [18] and seqinr [19] to read sequences and compute distance matrices, we preferred to avoid too many dependencies for clarity purpose. Thus the corresponding functions were rewritten and included into the bios $2 m d s$ package. Concerning the MDS computation, the $c m d s$ function provided by $\mathrm{R}$ gives the final results (coordinates and eigenvalues) but does not give access to the intermediary matrices that are required for the projection technique. It was thus necessary to write an MDS function from its basic equations in order to perform the projection of supplementary elements.

\section{Functionalities}

Here, we present the main functionalities of bios $2 m d s$. The package provides a complete $\mathrm{R}$ environment for MDS analysis in the context of protein sequences. It includes functions for data import, MDS computation, clustering and visualization of the results.

\section{Data import}

Multidimensional scaling relies on distance matrices. The user can provide these distance matrices or compute them from multiple sequence alignments in the FASTA or MSF formats. Sequence alignments are read in with the import.fasta or import.msf functions. Several measures of distances can be computed for multiple alignments of protein sequences: the Euclidean distances based on the square roots of the difference scores [20], the distances based on the difference scores and the distances based on dissimilarity scores computed from amino acid substitution matrices. The substitution matrices provided with bios $2 m d s$ are the JTT [21] and the Gonnet [22] matrices, the BLOSUM [23] and the PAM [24] series, along with the PHAT [25] and JTT_TM [26] matrices, more specifically developed for membrane proteins. The distances based on the difference scores can also be used for nucleotide sequences. More sophisticated measures of DNA distances can be found in several packages, such as ade4, ape, and phangorn $[14,18,27]$.

\section{MDS computation}

Briefly, given a matrix of (squared) distances between elements, MDS transforms this matrix of squared distances into a cross-product matrix whose eigendecomposition provides the factor score matrix giving the coordinates of the elements on the principal components [2-5]. The projection of supplementary elements onto the active space depends on the matrix of distances between the supplementary and the active elements and on the factor score matrix of the active elements $[2,12,13]$. To make the projection possible and to facilitate subsequent data analysis especially in the case of orthologous sequence sets, we provide the $m m d s$ function (corresponding to the cmds command from $\mathrm{R}$, but with a customized output) and the mmds.project function that performs projection of supplementary elements onto an active space.

\section{Clustering}

The MDS representation of the sequence space can be analyzed by $K$-means in order to find clusters. This part of bios $2 m d s$ depends on the amap [28], e1071 [29], and cluster [30] packages. The kmeans.run function that we have developed assesses the robustness of the clustering. It depends on the Kmeans and the matchClasses 
functions from the amap and e1071 packages, respectively. The kmeans.run function performs multiple runs of Kmeans from the amap package and analyzes the resulting clusters with the matchClasses function from the e1071 package. The output of kmeans.run provides the most frequent clustering solution, with the list of the members of each cluster and their relative membership to this cluster in the different runs. The sil.score function performs multiple runs of the silhouette function from the cluster package. The silhouette function helps determining the optimal number of clusters [31]. Multiple runs with sil.score allow taking into account the clustering uncertainty. The write.fasta function allows users to retrieve the multiple sequence alignment of each cluster, in FASTA format, for further analysis.

\section{Visualization}

The package bios $2 m d s$ contains graphical tools to visualize the results of the MDS analysis in $\mathrm{R}$. The mmds.2D.plot and mmds.3D.plot functions are used for $2 \mathrm{D}$ and $3 \mathrm{D}$ representations, respectively, of active and supplementary elements, on the same graph, on user selected components. The mmds.3D.plot function is based on plot $3 D$ from the $r g l$ package [32] that provides an interactive tool for $3 \mathrm{D}$ visualization within the $\mathrm{R}$ environment. The col.group function allows the use of a color code for plotting the $2 \mathrm{D}$ and $3 \mathrm{D}$ graphs, based on groupings and colors defined by the user in CSV files, for both active and supplementary elements. The mmds.2D.multi function visualizes the barycenters of the groups defined by the user in the $2 \mathrm{D}$ representation of the active elements. Finally, the write.mmds.pdb function allows users to export the coordinates of each element on the first three components obtained by MDS in a PDB format for visualization with a molecular graphics program, such as Pymol (www.pymol.org) or Rasmol (www.openrasmol.org). When col.group is used, each user-defined group corresponds to a different PDB chain number to facilitate selection and coloring.

\section{Results and discussion}

In this section, we show and discuss the results obtained by typical MDS analyses. The input consists of nonredundant sets of non-olfactory class A G-proteincoupled receptors (GPCRs) from different species [9,33]. Two types of analysis make sense: the analysis of paralogous sequences, yielding a sequence space, and the comparison of orthologous sequences, using the projection technique.

\section{Analysis of paralogous sequences}

The human set includes 283 aligned sequences of GPCRs [9]. The MDS analysis of this set provides a typical sequence space (Figure 1). In this example, the distances

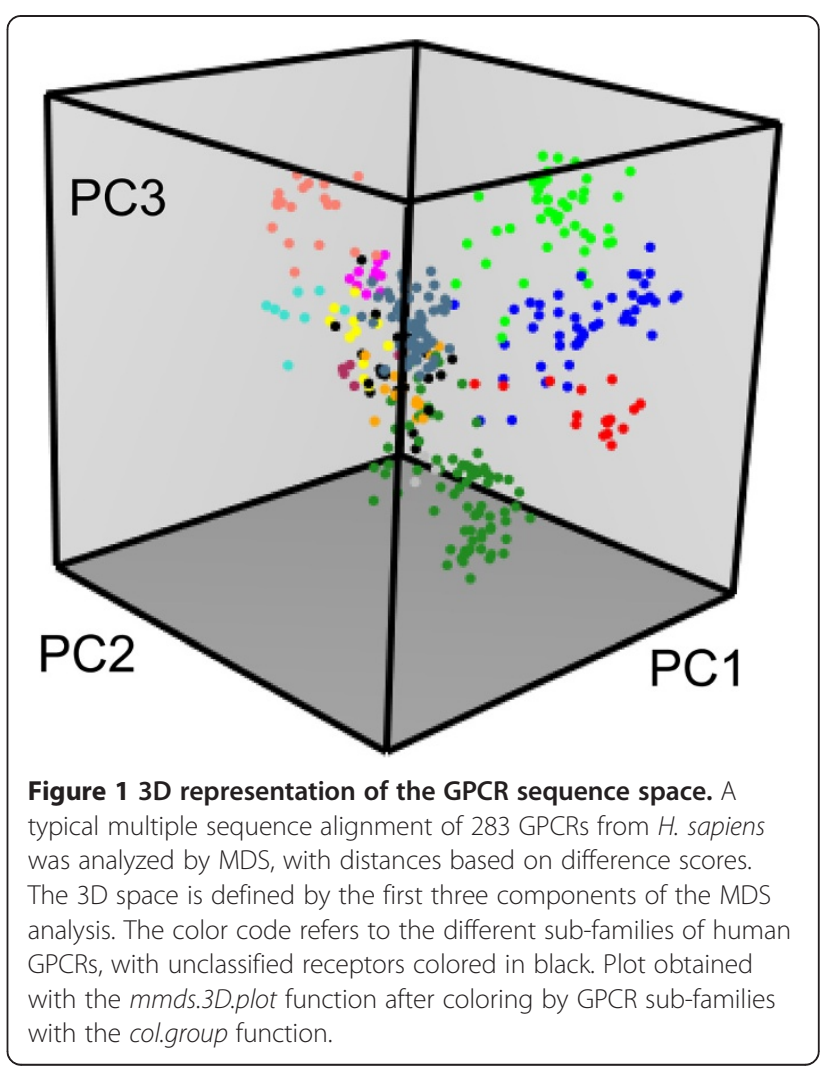

between sequences are equal to their difference scores and the 3D sequence space of human GPCRs is displayed with the plot $3 D$ command from the $r g l$ package [32] that allows interactive 3D representation within the $\mathrm{R}$ environment. The elements are colored using the color.group function based on the prior knowledge of the twelve GPCR sub-families present in humans $[9,33,34]$. Clustering allows the grouping of these sub-families into four groups that correspond to major pathways of GPCR evolution [9].

Different distance matrices can be computed from a multiple sequence alignment. These matrices are based either on a difference score or on a dissimilarity score obtained with an amino acid substitution matrix. In MDS, the distance matrix should be Euclidean or close to a Euclidean matrix. Distances equal to the square roots of the difference scores are Euclidean [20], and the MDS analysis of the corresponding matrix gives only positive eigenvalues. Distances equal to the difference scores give negative eigenvalues representing about 3\% of the variance, whereas distances based on dissimilarity scores give negative eigenvalues that can represent from 3 to $7 \%$ of the variance (Table 1).

The sequence spaces of human GPCRs obtained with the different distance matrices do not reveal dramatic differences and the overall patterns are maintained (Figure 2). In particular, the sequence spaces obtained 
Table 1 Comparison of scoring methods

\begin{tabular}{lll}
\hline Scoring method & & \% negative components \\
\hline Difference & Square root & 0 \\
Dissimilarity & Difference & 3.2 \\
& BLOSUM30 & 4.1 \\
& BLOSUM45 & 3.5 \\
BLOSUM62 & 3.6 \\
BLOSUM80 & 3.5 \\
PAM40 & 4.3 \\
PAM80 & 4.8 \\
PAM120 & 5.1 \\
PAM160 & 5.6 \\
PAM250 & 6.3 \\
GONNET & 3.5 \\
JTT & 6.5 \\
JTT_TM & 6.7 \\
PHAT & 4.1 \\
\hline
\end{tabular}

The percent of negative components represents the weight of negative components in the variance of the data. from the difference scores or their square roots are very similar and the slight changes observed with dissimilarity scores are quite independent of the matrix used for the computation. This is illustrated in Figures $2 \mathrm{c}$ and $\mathrm{d}$ that show the 2D sequence spaces obtained with the "best" and the "worst" matrices, as defined by comparison to Euclidean distances (Table 1)

The "noise" of the data can be estimated from the MDS analysis of a random sequence alignment (Figure 3) that is generated with the random function and has the same length, number of sequences, and amino acid composition as the initial alignment.

\section{Comparison of orthologous sequence sets}

In the example shown in Figure 4, the input consists of two sets of aligned sequences [9]: the set of 283 GPCR sequences from $H$. sapiens and a set of 538 GPCR sequences from $N$. vectensis (the sea anemone). Among these anemone sequences, 139 can be assigned to five sub-families present in humans. The mmds.project function projects the "supplementary" sequences onto the "active" sequence space. The positions of the supplementary elements depend only on their distances to active
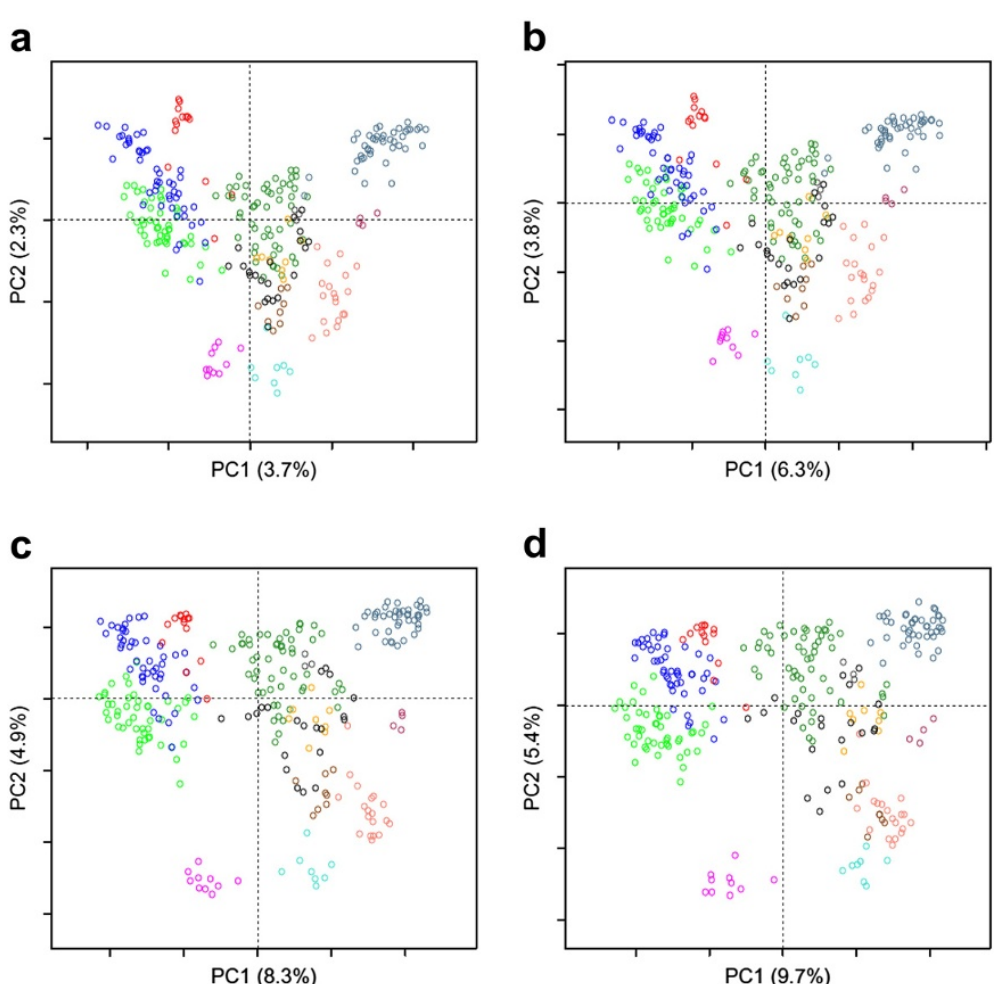

Figure 2 Comparison of scoring methods. The 2D sequence space of human GPCRs, defined by the first two components of the MDS analysis, was obtained with distances equal to the square roots of the difference scores (a), to the difference scores (b), to the dissimilarity scores calculated from the BLOSUM45 matrix (c) or from the JTT_TM matrix (d). The color code refers to the different sub-families of human GPCRs, with unclassified receptors colored in black. Plots drawn with the mmds.2D.plot function after coloring by GPCR sub-families with the col.group function. 


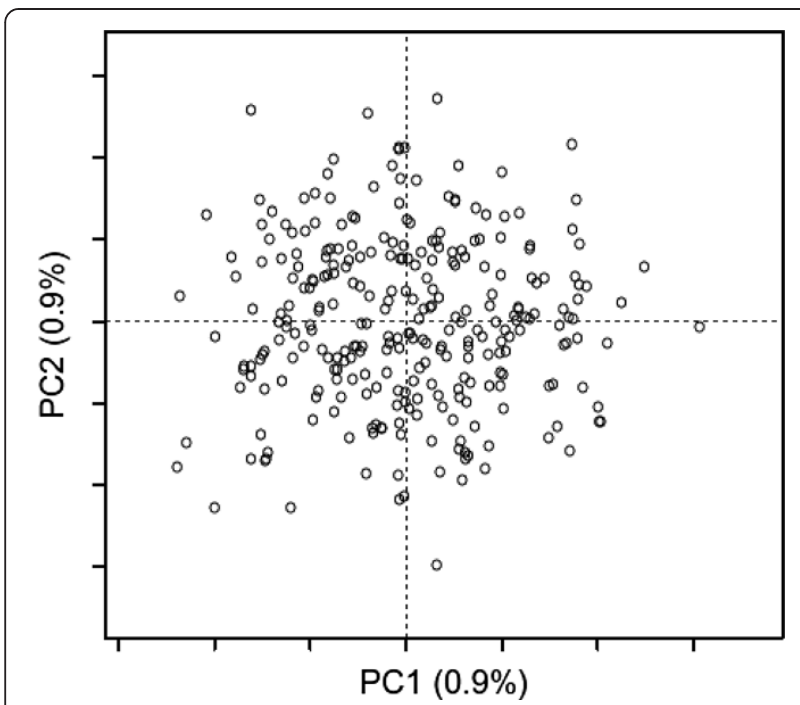

Figure 3 Sequence space of random sequences. The $2 \mathrm{D}$ sequence space of random sequences corresponds to the first two components of the MDS analysis of a random sequence alignment with the same properties as the human GPCR set, obtained with the random function. Plot drawn with the $m m d s$.2D.plot function. elements. Either set can be alternatively active or supplementary. By this way, we can analyze the GPCR evolution from the point of view of humans or of sea anemones.

Figures $4 \mathrm{a}$ and $\mathrm{b}$ show the projection of the assigned and unassigned sequences from $N$. vectensis, respectively, onto the sequence space of human GPCRs. As previously discussed [9], receptors that cannot be assigned to a human sub-family are projected onto the centre of the human space since their specific evolution is expected to happen on perpendicular dimensions. The GPCR sequence space of the sea anemone has a cross shape (Figure 4c) and is driven by several large sub-families with no equivalent in humans. For clarity purpose, we project separately the human GPCRs from either "ancient" or "recent" sub-families onto this sequence space (Figures $4 \mathrm{c}$ and $\mathrm{d}$, respectively). The "ancient" subfamilies are those present both in $N$. vectensis and in $H$. sapiens [9]. The projection of the human receptors from these "ancient" sub-families onto the anemone sequence space is consistent with the reverse projection (compare Figures $4 \mathrm{a}$ and c). Similarly, the human receptors from "recent" sub-families, with no equivalent in $N$. vectensis, are projected towards the centre of the anemone sequence space (Figure $4 \mathrm{~d}$ ).
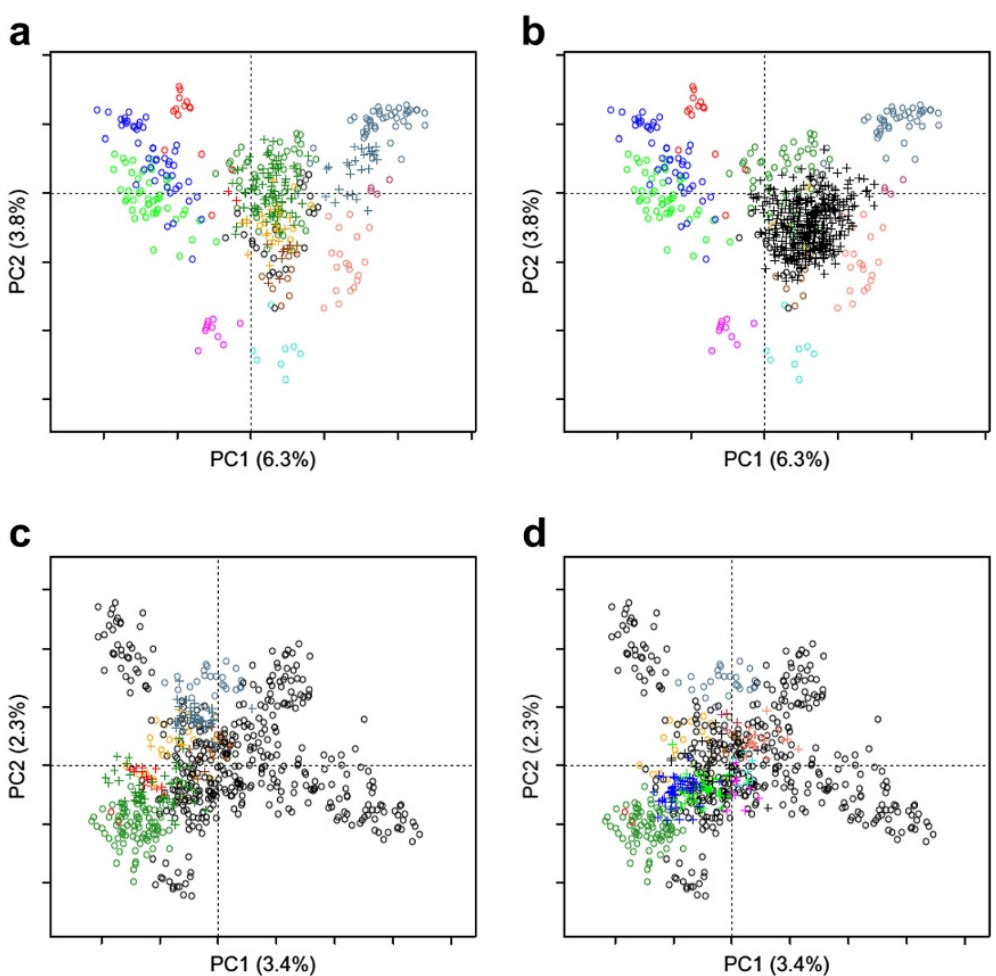

Figure 4 Projection of supplementary elements. Multiple sequence alignments of GPCRs from $\mathrm{H}$. sapiens and N. vectensis were analyzed by MDS, using either human GPCRs $(a, b)$ or anemone GPCRs $(c, d)$ as active sequences and the other set as supplementary sequences. The active and supplementary sequences are respectively represented by dots and crosses. They are projected onto the plane defined by the first two components of the active space. The color code refers to the different sub-families of humans GPCRs, with unclassified receptors colored in black. Plots drawn with the mmds.2D.plot function after coloring by GPCR sub-families with the col.group function. 


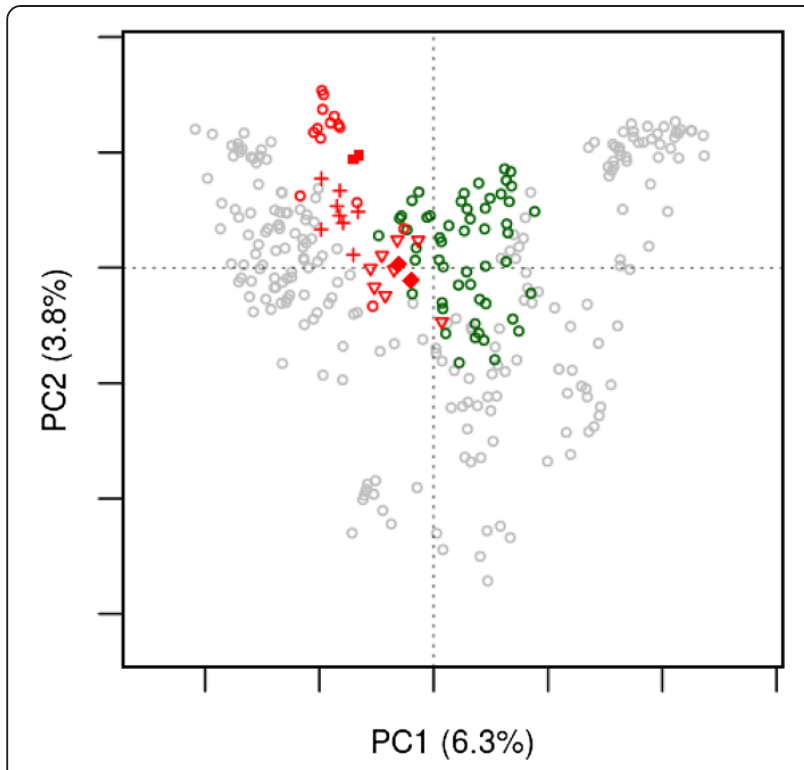

Figure $\mathbf{5}$ Evolutionary drift. The active sequence space (open circles) was obtained by the MDS analysis of the human GPCR set. Human receptors from the SO and PEP sub-families are indicated by red and green circles, respectively. The supplementary sequences correspond to SO receptors from $N$. vectensis (closed diamonds), $C$. elegans (open triangles), C. intestinalis (crosses) and D. melanogaster (closed squares). The active and supplementary sequences are projected onto the plane defined by the first two components of the active space. Plot drawn with the mmds.2D.plot function.

The usefulness of the projection technique is illustrated by the example of the somatostatin/opioid receptor sub-family (SO). The input consists of two sets of aligned sequences: the human set that includes $14 \mathrm{SO}$ receptors, and a set of receptors from $N$. vectensis, C. elegans, $C$. intestinalis and D. melanogaster that could be assigned to the SO sub-family [9,33]. Figure 5 shows the projection of the orthologous SO receptors onto the sequence space of human GPCRs. We have shown previously that this sub-family initiated from a deletion in an ancestor of the peptide receptors (PEP) that led to the split between these two sub-families [33]. The SO receptors from remote species ( $N$. vectensis and $C$. elegans) are close to the PEP receptors, whereas those from the chordate $C$. intestinalis and from the fruit fly D. melanogaster (the allatostatin receptors 1 and 2 [35]), are located at intermediary positions. Thus the projection technique reveals the evolutionary drift of specific subfamilies.

\section{Conclusions}

The R package bios $2 m d s$ provides users with a powerful and flexible framework to perform multidimensional scaling of multiple sequence alignments. The program can import data directly as distance matrices or as multiple sequence alignments from which distance matrices are computed. The package bios $2 m d s$ is tailored for the analysis of protein sequences but can be adapted for nucleotide sequences. Several $\mathrm{R}$ tools, including the $\mathrm{cmds}$ command, offer the possibility to perform MDS analysis of a distance matrix. However, to our knowledge, the bios $2 m d s$ package is unique in allowing the projection of supplementary elements onto an active space in the $\mathrm{R}$ environment. This property is especially suited for the comparison of orthologous sequence sets and the evolution of specific protein sub-families. The tools for the visualization of the data have been designed to take advantage of prior knowledge on the protein family under scrutiny, for example its classification into sub-families or the presence of specific sequence motifs. Finally, it has to be emphasized that the input of the projection function within bios $2 m d s$ requires only active and supplementary distances matrices. Thus this function can be widely used for any kind of application.

\section{Availability and requirements}

- Project name: bios2mds

- Project home page: http://cran.r-project.org/web/ packages/bios2mds/index.html

- Operating systems: Platform independent

- Programming language: R 2.12

- Other requirements: requires the amap, e1071, cluster, scales and rgl packages

- License: GNU General Public License

- Any restrictions to use by non-academics: None

\section{Competing interests}

The authors declare that they have no competing interests.

\section{Authors' contributions}

JP and JMB contributed equally to this work. JP and MC conceived the package. HA provided the projection method and support to implement it in R. JP and JMB wrote the software, which JP, MC and JMB tested and debugged. MC wrote the first draft of the manuscript, which HA revised and all authors approved.

\section{Acknowledgements}

We thank NEC Computers Services SARL (Angers, France) for the kind provision of a multiprocessor server. We thank the Conseil Général de Maineet-Loire for JP's fellowship and the Centre Hospitalier Universitaire of Angers and the CNRS for JMB's studenship. We thank Dr P. Guardiola (Angers, France) for stimulating discussion and advice.

\section{Author details}

${ }^{1}$ CNRS UMR 6214 - INSERM 1083, Faculté de Médecine, 3 rue Haute de Reculée, Angers 49045, France. ${ }^{2}$ The University of Texas at Dallas, School of Behavioral and Brain Sciences, 800 West Campbell Road, Richardson, TX 75080-3021, USA.

Received: 3 January 2012 Accepted: 24 May 2012

Published: 15 June 2012

\section{References}

1. B-Rao C, Majumdar KC: Reconstruction of phylogenetic relationships. J Biosci 1999, 24:121-137.

2. Abdi $\mathrm{H}$ : Metric multidimensional scaling. In Encyclopedia of Measurement and Statistics. Edited by Salkind NJ. Thousand Oaks (CA): Sage; 2007:598-605. 
3. Borg I, Groenen PJF: Modern multidimensional scaling. New York: Springer 2005.

4. Gower JC: A comparison of some methods of cluster analysis. Biometrics 1967, 23:623-637.

5. Torgerson WS: Theory and methods of scaling. New York: Wiley; 1958.

6. Blackshields G, Sievers F, Shi W, Wilm A, Higgins DG: Sequence embedding for fast construction of guide trees for multiple sequence alignment. Algorithms Mol Biol 2010, 5:21.

7. B-Rao C, Majumbar K: Map-like representation of phylogenetic relationships: Application to tilapiine fish. J Fish Biol 1998, 52:1199-1217.

8. Higgins DG: Sequence ordinations: a multivariate analysis approach to analysing large sequence data sets. Comput Appl Biosci 1992, 8:15-22.

9. Pelé J, Abdi H, Moreau M, Thybert D, Chabbert M: Multidimensional scaling reveals the main evolutionary pathways of class A G-protein-coupled receptors. PLoS One 2011, 6:e19094.

10. Shi W, Lei F, Zhu C, Sievers F, Higgins DG: A complete analysis of HA and NA genes of influenza A viruses. PLoS One 2010, 5:e14454

11. Woolley KJ, Athalye M: A use for principal coordinate analysis in the comparison of protein sequences. Biochem Biophys Res Commun 1986, 140:808-813.

12. Trosset MW, Pribe CE: The out-of-sample problem for classical multidimensional scaling. Computational statistics \& data analysis 2008, 52:4635-4642.

13. Gower JC: Adding a Point to Vector Diagrams in Multivariate Analysis. Biometrika 1968, 55:582-585.

14. Chessel D, Dufour AB, Thioulouse J: The ade4 package-l: One-table methods. $R$ news $2004,4: 5-10$.

15. Culhane AC, Thioulouse J, Perriere G, Higgins DG: MADE4: an R package for multivariate analysis of gene expression data. Bioinformatics 2005, 21:2789-2790

16. Jombart $\mathrm{T}$ : adegenet: a $\mathrm{R}$ package for the multivariate analysis of genetic markers. Bioinformatics 2008, 24:1403-1405.

17. Oksanen J, Blanchet FG, Kindt R, Legendre P, O'Hara RB, Simpson GL, Solymos P, Stevens MHH, Wagner H: Vegan: Community Ecology Package. http://cran.r-project.org/package=vegan.

18. Paradis E, Claude J, Strimmer K: APE: Analyses of Phylogenetics and Evolution in R language. Bioinformatics 2004, 20:289-290.

19. Charif D, Lobry JR: SeqinR 1.0-2: a contributed package to the $R$ project for statistical computing devoted to biological sequences retrieval and analysis. In Structural approaches to sequence evolution: Molecules, networks, populations. Edited by Bastolla U, Porto M, Roman HE, Vendruscolo M. New York: Springer Verlag; 2007:207-232.

20. Gower JC: A general coefficient of similarity and some of its properties. Biometrics 1971, 27:857-871.

21. Jones DT, Taylor WR, Thornton JM: The rapid generation of mutation data matrices from protein sequences. Comput Appl Biosci 1992, 8:275-282.

22. Gonnet $\mathrm{GH}$, Cohen MA, Benner SA: Exhaustive matching of the entire protein sequence database. Science 1992, 256:1443-1445.

23. Henikoff S, Henikoff JG: Amino acid substitution matrices from protein blocks. Proc Natl Acad Sci U S A 1992, 89:10915-10919.

24. Dayhoff $M O$, Schwartz RM, Orcutt BC: A model of evolutionary change in proteins. Atlas of Protein Sequence and Structure 1978, 5:345-352.

25. Ng PC, Henikoff JG, Henikoff S: PHAT: a transmembrane-specific substitution matrix. Predicted hydrophobic and transmembrane. Bioinformatics 2000, 16:760-766

26. Jones DT, Taylor WR, Thornton JM: A mutation data matrix for transmembrane proteins. FEBS Lett 1994, 339:269-275.

27. Schliep KP: Phangorn: phylogenetic analysis in R. Bioinformatics 2011 , 27:592-593.

28. Lucas A: Using amap and ctc Packages for Huge Clustering. $R$ news 2006 6:58-60.

29. Dimitriadou E, Hornik K, Leisch F, Meyer D, Weingessel A: Misc Functions of the Department of Statistics (e1071), TU Wien. http://cran.r-project.org/ package $=\mathrm{e} 1071$

30. Struyf A, Hubert M, Rousseeuw PJ: Integrating Robust Clustering Techniques in S-PLUS. Computational Statistics and Data Analysis 1997, 26:17-37.

31. Rousseeuw P: Silhouettes: A Graphical Aid to the Interpretation and Validation of Cluster Analysis. J Comput Appl Math 1987, 20:53-65.

32. Adler D, Nenadic O, Zucchini W: RGL: A R-library for 3D visualization with OpenGL. In Proceedings of the 35th Symposium of the Interface: Computing
Science and Statistics: 12-15 March 2003; Salt Lake City. Edited by Braverman A. Red Hook: Curran Associates, Inc; 2009:419-429.

33. Devillé J, Rey J, Chabbert M: An indel in transmembrane helix 2 helps to trace the molecular evolution of class A G-protein-coupled receptors. J Mol Evol 2009, 68:475-489.

34. Fredriksson R, Lagerstrom MC, Lundin LG, Schioth HB: The G-proteincoupled receptors in the human genome form five main families. Phylogenetic analysis, paralogon groups, and fingerprints. Mol Pharmacol 2003, 63:1256-1272

35. Kreienkamp HJ, Larusson HJ, Witte I, Roeder T, Birgul N, Honck HH, Harder S, Ellinghausen G, Buck F, Richter D: Functional annotation of two orphan Gprotein-coupled receptors, Drostar1 and -2, from Drosophila melanogaster and their ligands by reverse pharmacology. J Biol Chem 2002, 277:39937-39943.

doi:10.1186/1471-2105-13-133

Cite this article as: Pelé et al:: Bios2mds: an R package for comparing orthologous protein families by metric multidimensional scaling. BMC Bioinformatics 2012 13:133.

\section{Submit your next manuscript to BioMed Central and take full advantage of:}

- Convenient online submission

- Thorough peer review

- No space constraints or color figure charges

- Immediate publication on acceptance

- Inclusion in PubMed, CAS, Scopus and Google Scholar

- Research which is freely available for redistribution 